\title{
Magnetic structure of almandine
}

\author{
D. Zherebetskyy, S. Lebernegg, G. Amthauer and M. Grodzicki \\ Dept. of Materials Research and Physics \\ University of Salzburg, Austria
}

The magnetic structure of almandine has been investigated by electronic structure calculations in the local spin density approximation in order to arrive at a more detailed understanding of the magnetic properties and the exchange pathways. The calculations are based on experimentally determined geometrical data of the crystal structure at $100 \mathrm{~K}$. The calculated quadrupolesplittings, spin-allowed $d$ $d$ transitions and magnetic moment for iron atoms are in reasonable agreement with the respective experimental values obtained by Mössbauer andabsorption spectroscopy, and by magnetization measurementsdemonstrating the reliability of the calculations. The spin structure is derived from the calculated magnetic coupling constants for the possible exchange pathways. Competing superexchange pathways exist via the oxygen bridges between iron ions of edge-sharing dodecahedra, and via edges of silicon tetrahedra and aluminium octahedra connecting more distant dodecahedra, respectively. The coupling between the iron spins of neighboured dodecahedra is always ferromagnetic, and ten of these dodecahedra form a ring of ferromagnetically coupled spins. The overall magnetic structure consists of mutually penetratingrings of dodecahedra connected via $\mathrm{Al}$ octahedra and Si tetrahedra. The coupling between two rings takes place via edges of $\mathrm{Al}$ octahedra and $\mathrm{Si}$ tetrahedraand is antiferromagnetic. In summary, the calculations reveal that almandine contains two identical magnetic sublatticesthat are coupled by weak antiferromagneticinteractions in qualitative agreement with the low Néel temperature of about $7 \mathrm{~K}$. Moreover, the calculated hyperfine parameters explain the Mössbauer spectra of almandine below the Néel temperature. 